\title{
A Theory for Building NEO-Classical Production Functions
}

\author{
Oscar Orellana ${ }^{1, *}$, Raúl Fuentes ${ }^{2}$ \\ ${ }^{1}$ Department of Mathematics, Federico Santa María Technical University, Chile \\ ${ }^{2}$ Department of Industries, Federico Santa María Technical University, Chile \\ Received October 17, 2021; Revised January 14, 2022; Accepted February 8, 2022
}

\section{Cite This Paper in the following Citation Styles}

(a): [1] Oscar Orellana, Raúl Fuentes , "A Theory for Building NEO-Classical Production Functions," Advances in Economics and Business, Vol. 10, No. 1, pp. 1 - 13, 2022. DOI: 10.13189/aeb.2022.100101.

(b): Oscar Orellana, Raúl Fuentes (2022). A Theory for Building NEO-Classical Production Functions. Advances in Economics and Business, 10(1), 1 - 13. DOI: 10.13189/aeb.2022.100101.

Copyright $\mathrm{C} 2022$ by authors, all rights reserved. Authors agree that this article remains permanently open access under the terms of the Creative Commons Attribution License 4.0 International License

\begin{abstract}
In this study, we propose a mathematical theory for building neoclassical production functions with homogeneous inputs in both aggregate and per capita terms. This theory is based on two concepts: Euler's equation and Cauchy's condition for first-order partial differential equations. The analysis is restricted to functions that exhibit constant returns to scale (CRS). For the function to meet the law of diminishing marginal returns, we present the necessary and sufficient conditions to be satisfied by the curve that defines Cauchy's condition. In this context, we also discuss the Inada conditions. We first present functions that depend on two inputs and then extend and discuss the results for functions that depend on several inputs. The main result of our research is the provision of a clean and clear theory for constructing neo-classical production functions. We believe that this result may contribute to closing the huge methodological gaps that separate schools of economic thought that defend or reject the use of production functions in economics.
\end{abstract}

Keywords NEO-Classical Production Functions, Partial Differential Equations, Euler's Equation, Cauchy's Condition, Homogeneous Inputs

JEL Classification: C02; E13

\section{Introduction}

Since the famous Cambridge capital controversy ${ }^{1}$, the

1 We refer to the capital theory and production function controversy that took place among the most important economists from the universities of Cambridge (UK) and Cambridge (Massachusetts, USA) production function in relation to the aggregation problem usually found in macroeconomics has been a topic of theoretical and empirical discussion (or debate) in the field of economics. Whereas prominent researchers such as Robert Solow, Paul Samuelson, and Franco Modigliani have defended economic orthodoxy (neoclassical synthesis), other researchers, including Nicholas Kaldor, Joan Robinson, Piero Sraffa, Richard Khan, and Pierangelo Garegnani, among others, are advocates of heterodoxy (post Keynesianism). To date, there has been no agreed upon result of this debate in relation to our study. Instead, two almost divergent agendas (schools of economic thought) that assign a very different role to the production function in their respective methodologies have resulted. From the orthodox perspective, the production function is a cornerstone of its methodological construct. For heterodoxy, its use is generally avoided. At this point, it is important to mention that part of the economic heterodoxy inherited from the Cambridge controversy categorically rejects both the theoretical and empirical possibility of the existence (use) of the production function, particularly, the aggregated production function. Theoretically, supporters of this position assert that it is impossible to overcome the dimensions, measures, and switching and reswitching problems of capital identified by Robinson and her colleagues during the Cambridge debate. Empirically, it is alluded that production functions in a macroeconometric analysis hide a simple accounting identity. This type of criticism originated with Shaik's (1974) provocative article, which has been extended more recently by J. Felipe and

that was initiated by Joan Robinson's famous article, "The production function and the theory of capital" (1953-1954). 
J.S.L. McCombie (2014), among others ${ }^{2}$. From an economic orthodoxy point of view, it is well known that the production function is one of the key concepts of mainstream neoclassical theories. The theory of production function is even a substantial part of both pre- and postgraduate micro- and macroeconomics courses. The Cobb-Douglas function, which was inspired by the empirical work of these authors during the 1920s, is by far the most widely used theoretical and empirical neoclassical production function in practice ${ }^{3}$. The CES function, which was introduced to economics by Arrow, Chenery, Minhas and Solow (1961), is well-reputed but less used in neoclassical economic practice ${ }^{4}$. In contrast, some variable elasticity of substitution (VES) production functions have emerged, but their use has remained quite sparse in theoretical and empirical literature ${ }^{5}$. The use of neoclassical production functions in economics is very diverse, from uses in microeconomics to macroeconomics. They have been widely used in microeconomic to study, among other things, a firm's short term and long term behaviors, and are a cornerstone of the whole theory of economic growth, as well as of the theory of international trade, in macroeconomics ${ }^{6}$.

However, in our opinion, all of these theories suffer from an important declivity: they presume the theoretical existence of the production function. Once this function is given, different properties and characteristics are studied. This presumption opens a very large space to explore the conditions that are required to generate production functions, which is precisely the objective of our research agenda. Given the ambitious nature of this agenda, in this article, we search for the minimum conditions required for a mathematical formulation in the form $Y=$ $F\left(X_{1}, X_{2}, \cdots, X_{n}, L\right)$ to be considered a neoclassical production function. In other words, a formulation that satisfies the properties of constant returns to scale (CRS) and the law of diminishing marginal returns. To achieve the stated objective, we propose a three-step methodology restricted to the analysis of homogenous inputs. First, we define what we call a protoproduction function, that is, a two-input function that satisfies both Euler's equation (EE) and Cauchy's condition. This function must first comply with the CRS property, which is very usual and easy to interpret in economic terms. To the best of our knowledge, the second requirement is not used in the current theory of production functions. In this context, we postulate that Cauchy's condition represents the necessary economic

2 The link of this identity has a crystalline demonstration in the case of the Cobb-Douglas and constant elasticity of substitution (CES) functions. 3 See Cobb and Douglas (1928). We also suggest reading the recent work by Biddle (2010) for a historical and modern perspective of this function. 4 The acronym CES represents the constant elasticity of substitution between factors. While the Cobb-Douglas function assumes an elasticity of substitution between productive factors equal to 1, the CES function allows values that differ from 1 . See Mishra (2007) for more technical and historical details on the CES production function.

5 See for example Revankar (1971), Karagiannis et al. (2005) and Tach (2020).

6 For readers unfamiliar with this subject, it is highly recommended to visit the works by Barro and Sala-i-Martin (2003), Mas-Colell, Whinston and Green (1995) and Krugman and Obstfeld (2002). data in the form of a time series, which is used to parametrize the corresponding production function ${ }^{7}$. Once the protoproduction function is realized, the necessary and sufficient conditions to satisfy the law of diminishing marginal returns are obtained. In terms of noncompliance, we inquire into the conditions under which the production function built up to this stage satisfies the well-known Inada conditions, which are the usual properties required for the formulation of per capita production functions. Once this objective is achieved, the objective of formulating a two-factor neoclassical production function is also met. Finally, we extend this theory to more than two production factors. In each step, we complement our theory with examples.

To clarify, in our article, we do not attempt to support one economic vision or the other. However, it is clear that, in part, our work begins to challenge the position of Shaik and his successors, which denies the theoretical possibility of the existence of production functions. From a strictly mathematical point of view (which should not be confused with the practical and empirical points of view), it is worth mentioning that our theory is well posed in the sense of Hadamard. That is, (a) there is a solution, (b) the solution is unique, and (c) the solution continuously depends on the data (in our case from Cauchy's condition).

The remainder of this paper is given as follows. In Sections II and III, we present the theory for obtaining protoproduction functions for two inputs. In Section IV, we obtain the necessary and sufficient conditions required for these functions to satisfy the law of diminishing marginal returns. In Section V, we present and discuss the relationship between the self-similar solution and the per capita production function and Inada conditions. In Section VI, we generalize the theory to several independent and homogeneous production factors. Section VII concludes this work.

\section{Basic Definitions}

In this section, we present, develop, and discuss a mathematical theory of the protoproduction functions capable of being represented in the form $Y=$ $F\left(X_{1}, X_{2}, \cdots, X_{n}, L\right)$. This function satisfies both $\mathrm{EE}$ and Cauchy's condition. As applied to economics, EE is equivalent to the property of CRS. Lesser known in economics, Cauchy's condition for first-order partial differential equations is given by:

$\gamma\left(\tau_{1}, \tau_{2}, \ldots, \tau_{n}\right)$
$=\mathrm{F}\left(\alpha_{1}\left(\tau_{1}, \cdots, \tau_{n}\right), \cdots, \alpha_{n}\left(\tau_{1}, \cdots, \tau_{n}\right), \beta\left(\tau_{1}, \cdots, \tau_{n}\right)\right)$

The economic intuition underlying Cauchy's condition is as follows. Let us consider production functions that depend only on two factors: capital (K) and labor (L). Then,

7 Additionally, we will show that from this condition we can build theoretical support for the problem of aggregation found in macroeconomics, which is a very attractive result of our theory. 
assume the production activity of a particular industry, sector or country can be represented by a production function in the form $\mathrm{Y}=\mathrm{F}(\mathrm{K}, \mathrm{L})$. Cauchy's condition is reduced to $\gamma(\tau)=F(\alpha(\tau), \beta(\tau))$, where $\alpha(\tau), \beta(\tau)$ and $\gamma(\tau)$ are functions of the unique parameter $\tau \in[0, T]$ (instead of $n$-parameters $\left.\tau_{1}, \tau_{2}, \cdots, \tau_{n} 8\right)$. Hence, if we interpret $\tau$ as time, then $\alpha(\tau), \beta(\tau)$ and $\gamma(\tau)$ can be interpreted as continuous time series representations of capital, labor and output data, respectively, of the given industry, sector or country. The production function $Y=F(K, L)$, which is a function of the reduced form of Cauchy's condition $\gamma(\tau)=F(\alpha(\tau), \beta(\tau))$ of two independent production factors, must go through the 3-dimensional data curve $\alpha(\tau), \beta(\tau), \gamma(\tau): \tau \in$ $[0, T]$ obtained from the given production activity. In other words, the corresponding production function that represents the given production activity must fully absorb the data curve associated with such production activity. The problem of obtaining the continuous time series representations of capital, labor and output is a matter of econometric and curve adjustments. We make some comments on this in the next sections of this paper. For now, it is important to remark that if the following determinant of a Jacobian matrix:

$J=\left|\begin{array}{ccccc}\frac{\partial \alpha_{1}}{\partial \tau_{1}} & \frac{\partial \alpha_{2}}{\partial \tau_{1}} & \cdots & \frac{\partial \alpha_{n}}{\partial \tau_{1}} & \frac{\partial \beta}{\partial \tau_{1}} \\ \frac{\partial \alpha_{1}}{\partial \tau_{2}} & \frac{\partial \alpha_{2}}{\partial \tau_{2}} & & \frac{\partial \alpha_{n}}{\partial \tau_{2}} & \frac{\partial \beta}{\partial \tau_{2}} \\ \vdots & \vdots & \cdots & \vdots & \vdots \\ \frac{\partial \alpha_{1}}{\partial \tau_{n}} & \frac{\partial \alpha_{2}}{\partial \tau_{n}} & & \frac{\partial \alpha_{n}}{\partial \tau_{n}} & \frac{\partial \beta}{\partial \tau_{n}} \\ \alpha_{1}\left(\tau_{1}, \cdots, \tau_{n}\right) & \alpha_{2}\left(\tau_{1}, \cdots, \tau_{n}\right) & \ldots & \alpha_{n}\left(\tau_{1}, \cdots, \tau_{n}\right) & \beta\left(\tau_{1}, \cdots, \tau_{n}\right)\end{array}\right|$

differs from zero, then the Cauchy problem for EE has a unique solution. Moreover, the solution depends continuously on the data manifold given parametrically by:

$\vec{r}=\vec{r}\left(\tau_{1}, \cdots, \tau_{n}\right)$

$=\left(\alpha_{1}\left(\tau_{1}, \cdots, \tau_{n}\right), \cdots, \alpha_{n}\left(\tau_{1}, \cdots, \tau_{n}\right), \beta\left(\tau_{1}, \cdots, \tau_{n}\right), \gamma\left(\tau_{1}, \cdots, \tau_{n}\right)\right)$

which reduces to $\vec{r}=\vec{r}(\tau)=(\alpha(\tau), \beta(\tau), \gamma(\tau))$ in the case of two production factors. Therefore, under the hypothesis that $J \neq 0$, Cauchy's problem for EE is a well-posed problem in the sense of Hadamard ${ }^{9}$. Hence, these are the logical mathematical conditions (abstract and ideal) for a theory of the 'homogeneity of degree one' (HDO) protoproduction functions.

Moreover, given the solution of the Cauchy problem for EE under the assumption that $J \neq 0$, we deduce the additional conditions that the protoproduction function must satisfy to comply with the law of diminishing marginal returns. As may be expected, these conditions

8 In this context, $T$ represents the period of time in which the historical time series data is registered.

9 The mathematical term "well-posed problem" stems from a definition given by Jacques Hadamard. He believed that mathematical models of physical phenomena should have the following properties: a solution exists, the solution is unique, and the solution's behavior changes continuously with the initial conditions. fall on the manifold data given parametrically by $\vec{r}=$ $\vec{r}\left(\tau_{1}, \tau_{2}, \cdots, \tau_{n}\right)$ above. From a purely theoretical point of view, this theory works well, and we present some examples. However, from a practical point of view, we now visualize that there are serious empirical difficulties in applying this theory in practice, except for production activities that depend on only two independent and homogeneous production factors. One epistemologically interesting consequence of the theory proposed in this study is that it allows us to define the protoproduction function of a given production activity with CRS as one of the two following equivalent forms:

Definition II.1. Given that $J \neq 0$, a function of the form $Y=F\left(X_{1}, X_{2}, \cdots, X_{n}, L\right)$ is a proto-production function if it has a parametric representation of the form. Then,

$$
\begin{gathered}
\left.X_{1}=X_{1}\left(\tau_{1}, \tau_{2}, \cdots, \tau_{n}, t\right)\right)=\alpha_{1}\left(\tau_{1}, \tau_{2}, \cdots, \tau_{n}\right) e^{t} \\
\left.X_{2}=X_{2}\left(\tau_{1}, \tau_{2}, \cdots, \tau_{n}, t\right)\right)=\alpha_{2}\left(\tau_{1}, \tau_{2}, \cdots, \tau_{n}\right) e^{t} \\
\vdots \\
\left.X_{n}=X_{n}\left(\tau_{1}, \tau_{2}, \cdots, \tau_{n}, t\right)\right)=\alpha_{n}\left(\tau_{1}, \tau_{2}, \cdots, \tau_{n}\right) e^{t} \\
\left.L=L\left(\tau_{1}, \tau_{2}, \cdots, \tau_{n}, t\right)\right)=\beta\left(\tau_{1}, \tau_{2}, \cdots, \tau_{n}\right) e^{t} \\
\left.Y=Y\left(\tau_{1}, \tau_{2}, \cdots, \tau_{n}, t\right)\right)=\gamma\left(\tau_{1}, \tau_{2}, \cdots, \tau_{n}\right) e^{t}
\end{gathered}
$$

where $\left\{X_{1}, X_{2}, \cdots, X_{n}, L\right\}$ are the production input factors, $Y$ is the production output, and $\left(\alpha_{1}, \alpha_{2}, \cdots, \alpha_{n}, \beta, \gamma\right)\left(\tau_{1}, \tau_{2}, \cdots, \tau_{n}\right)$ is the parametric representation of an n-dimensional manifold that represents the empirical data of a given production activity.

Definition II.2. Given that $J \neq 0$, a function of the form $Y=F\left(X_{1}, X_{2}, \cdots, X_{n}, L\right)$ will be called a protoproduction function if it satisfies the following conditions:

$$
X_{1} \frac{\partial F}{\partial X_{1}}+X_{2} \frac{\partial F}{\partial X_{2}}+\cdots+X_{n} \frac{\partial F}{\partial X_{n}}+L \frac{\partial F}{\partial L}=F
$$

$\gamma\left(\tau_{1}, \tau_{2}, \cdots, \tau_{n}\right)$

$=F\left(\alpha_{1}\left(\tau_{1}, \tau_{2}, \cdots, \tau_{n}\right), \cdots, \alpha_{n}\left(\tau_{1}, \tau_{2}, \cdots, \tau_{n}\right), \beta\left(\tau_{1}, \tau\right.\right.$

From (II.1), we can determine the corresponding restrictions that $\alpha_{1}, \alpha_{2}, \cdots, \alpha_{n}, \beta$, and their respective first and second derivatives must meet so that the protoproduction function satisfies the law of diminishing marginal returns. Additionally, because $J \neq 0$, it follows that the first $(n+1)$ relations of (II.1) are locally invertible. Therefore, (II.1) is the parametric representation of a function in the form $Y=F\left(X_{1}, X_{2}, \cdots, X_{n}, L\right)$. Another interesting consequence of this theory is that because of Cauchy's condition (II.3), the problem of aggregation found in macroeconomics is given some theoretical support.

\section{Euler's Theorem and Some Economic Consequences}

Consider a function in the form $Y=F(K, L)$, where $Y$ is the dependent variable that represents the total production (output), and $K$ and $L$ are the independent variables that 
represent capital and labor, respectively. From a mathematical point of view, $K, L$, and $Y$ are continuous homogeneous variables. More precisely, we begin with a continuous differentiable function represented by:

$$
\begin{gathered}
F: \quad N \subseteq \mathbb{R}^{+} \times \mathbb{R}^{+} \rightarrow \mathbb{R}_{0}^{+} \\
(K, L) \rightarrow Y=F(K, L)
\end{gathered}
$$

where $N$ is the appropriate domain of $Y=F(K, L)$. As is well known in micro- and macroeconomics, one of the very usual properties attributable to (III.1) is that it satisfies the so-called CRS condition. This means that the function (III.1) has to be HDO, namely:

$$
F(\lambda K, \lambda L)=\lambda F(K, L)=\lambda Y
$$

which can be proven to be equivalent to the first-order linear partial differential equation known as EE. Hence, under our assumptions we have: $(C R S) \Leftrightarrow(H D O) \Leftrightarrow$ $(E E)$. The proof of Euler's theorem is well known and requires no further details here. More importantly, for our purposes, from Euler's theorem, we have two a priori alternatives to solve the problem of determining the function $Y=F(K, L)$. The first strategy (the standard method) is to solve the 'Cauchy problem of EE', which, in formal terms, is set as follows:

$$
\left\{\begin{array}{c}
K \frac{\partial F}{\partial K}+L \frac{\partial F}{\partial L}=F, \text { with } Y=F(K, L), \text { and such that } \\
\gamma(\tau)=F(\alpha(\tau), \beta(\tau)) \text { when } \tau \in[0, T) \text {, and where } \\
\vec{r}=\vec{r}(\tau)=(\alpha(\tau), \beta(\tau), \gamma(\tau)) \\
\text { is the parametization of space curve. }
\end{array}\right.
$$

The second strategy is to solve EE requiring additional properties for its solution. For example, $\mathrm{Y}=\mathrm{F}(\mathrm{K}, \mathrm{L})$ satisfies the law of diminishing marginal returns for each factor of production separately. In this case, the problem can be stated as follows:

$$
\left\{\begin{aligned}
& K \frac{\partial F}{\partial K}+L \frac{\partial F}{\partial L}=F, \text { where } Y=F(K, L), \\
& \text { and such that } \\
& \frac{\partial F}{\partial K}>0, \frac{\partial F}{\partial L}>0, \frac{\partial^{2} F}{\partial K^{2}}<0, \text { and } \frac{\partial^{2} F}{\partial L^{2}}<0
\end{aligned}\right.
$$

However, problems (III.3) and (III.4) are compatible. In fact, in section III it is shown that the solution of problem (III.3) can satisfy (for example) the law of diminishing marginal returns if the component of the parametric curve $\vec{r}=\vec{r}(\tau)=(\alpha(\tau), \beta(\tau), \gamma(\tau))$ and its first and second derivatives satisfy certain conditions. Next, we solve problem (III.3) by following the standard mathematical method (see [1]) and make some comments. Let us first assume that the integral surface (production function) $Y=F(K, L)$ can be represented parametrically by $K=K(\tau, t) ; L=L(\tau, t) ; Y=Y(\tau, t)$, where $\tau$ and $\mathrm{t}$ are only parameters for the time being. However, $\tau \in[0, T)$. Using a geometrical interpretation of the first-order partial differential equation, it can be proven (under suitable smooth assumptions) that the integral surface $Y=F(K, L)$ is composed of the so-called characteristic curves that satisfy the following system of ordinary differential equations and initial conditions:

$$
\left\{\begin{array}{l}
\frac{d F}{d t}=K(\tau, t) \text { such that } K(\tau, 0)=\alpha(\tau) \\
\frac{d L}{d t}=L(\tau, t) \text { such that } L(\tau, 0)=\beta(\tau) \\
\frac{d Y}{d t}=Y(\tau, t) \text { such that } Y(\tau, 0)=\gamma(\tau)
\end{array}\right.
$$

By integrating (III.5), we obtain:

$$
\left\{\begin{array}{l}
K=K(\tau, t)=\alpha(\tau) e^{t} \\
L=L(\tau, t)=\beta(\tau) e^{t} \quad \tau \in[0, T] \\
Y=Y(\tau, t)=\gamma(\tau) e^{t}
\end{array}\right.
$$

From the implicit function theorem (for two equations), it follows that if the determinant of the Jacobian matrix: $\left|\frac{\partial(K, L)}{\partial(\tau, t)}\right|_{\left(\tau_{0}, 0\right)}$ differs from zero, then the first two equations in (III.6) are locally invertible and $\tau=\tau(K, L)$ and $t=t(K, L)$ are defined in the neighborhood of the operation point: $\left(K_{0}, L_{0}, Y_{0}\right)=\left(\alpha\left(\tau_{0}\right), \beta\left(\tau_{0}\right), \gamma\left(\tau_{0}\right)\right)$ for some $\left.\tau_{0} \in[0, T]\right]$, where $K_{0}=K\left(\tau_{0}, 0\right)$, $L_{0}=L\left(\tau_{0}, 0\right)$, and $Y_{0}=Y\left(\tau_{0}, 0\right)$.

For problem (III.3) and considering (III.5), the condition

$\left|\frac{\partial(K, L)}{\partial(\tau, t)}\right|_{\left(\tau_{0}, 0\right)} \neq 0$ reduces to:

$$
\begin{aligned}
J & =\operatorname{det}\left(\begin{array}{ll}
\frac{\partial K}{\partial \tau}\left(\tau_{0}, 0\right) & \frac{\partial K}{\partial \tau}\left(\tau_{0}, 0\right) \\
\frac{\partial L}{\partial \tau}\left(\tau_{0}, 0\right) & \frac{\partial L}{\partial \tau}\left(\tau_{0}, 0\right)
\end{array}\right)=\left|\begin{array}{ll}
\alpha^{\prime}\left(\tau_{0}\right) & K\left(\tau_{0}\right) \\
\beta^{\prime}\left(\tau_{0}\right) & L\left(\tau_{0}\right)
\end{array}\right| \\
& =\alpha^{\prime}\left(\tau_{0}\right) L\left(\tau_{0}\right)-\beta^{\prime}\left(\tau_{0}\right) K\left(\tau_{0}\right)
\end{aligned}
$$

From (III.7), it follows that EE at the origin $(K, L)=$ $(0,0)$ is singular (i.e., $J=0)$. This means that in the neighborhood of the origin $(K, L)=(0,0)$, we cannot invert the relations $K=K(\tau, t)$ and $L=L(\tau, t)$; therefore, there is no integral surface of the form $Y=$ $F(K, L)$ in the neighborhood of the origin $(K, L)=(0,0)$. However, if the projection curve $\vec{l}=\vec{l}(\tau)=$ $(\alpha(\tau), \beta(\tau))$ does not pass through the origin $(K, L)=(0,0)$, condition (III.7) guarantees the existence and uniqueness of the solution of problem (III.3) in the neighborhood of the point of operation $P_{0}=\left(K_{0}, L_{0}, Y_{0}\right)=$ $\left(\alpha\left(\tau_{0}\right), \beta\left(\tau_{0}\right), \gamma\left(\tau_{0}\right)\right)$. Geometrically, problem (III.3) has a unique solution in the neighborhood of the operation point $P_{0}=\left(K_{0}, L_{0}, Y_{0}\right)=\left(\alpha\left(\tau_{0}\right), \beta\left(\tau_{0}\right), \gamma\left(\tau_{0}\right)\right)$ if the curve $\mathrm{P}$, defined by $\vec{r}=\vec{r}(\tau)=(\alpha(\tau), \beta(\tau), \gamma(\tau))$, is transversal to the characteristic curves that compose the integral surface (production function) $Y=F(K, L)$ or the 
planar curve $\vec{l}=\vec{l}(\tau)=(\alpha(\tau), \beta(\tau))$ does not coincide with one of the projections of the characteristic curves in the K-L plane that form the integral surface $Y=F(K, L)$. The projection of the characteristic curves in the $\mathrm{K}-\mathrm{L}$ plane are called characteristic projections and should not be confused with the characteristic curves because in our problem, the characteristic curves are three-dimensional curves, whereas the characteristic projections of these curves are two-dimensional curves. Note that the characteristic projections of problem (III.3) can easily be obtained by integrating the ordinary differential equation:

$$
\frac{\partial L}{\partial K}=\frac{L}{K}
$$

yielding $L=\sigma K$, where is an arbitrary constant of integration. Note that $L=\sigma K$ is a straight line that springs from the origin. Therefore, to obtain a unique solution to problem (III.3), we have to ensure that the two-dimensional data curve $\vec{l}(\tau)=(\alpha(\tau), \beta(\tau))$ does not coincide with one of the straight lines $L=\sigma K$ (i.e., $\vec{l}=\vec{l}(\tau)=(\alpha(\tau), \beta(\tau))$ has to be transversal to the straight line $L=\sigma K)$.

From an economic point of view, both the existence and uniqueness of the production function are important. Obviously, given a production activity, the first task is to establish that it has a production function associated with it; otherwise, it is illogical to attempt to find its production function. In contrast, it is illogical to consider that a given production activity has more than one production function associated with it. In other words, if a production activity has a production function, it must be unique. From a practical point of view, it is probably difficult (if not impossible) to argue the existence of the production function. Hence, to remain clear, we consider the theoretical existence and uniqueness of the production function, using EE and Cauchy's condition as basic components. Therefore, from a mathematical point of view, given (III.7), problem (III.3) is well posed according to Hadamard, as described above. At this point, it is worth mentioning that, to the best of our knowledge, specialists in production functions and related themes never explicitly impose Cauchy's condition on these functions, probably because there is no economic interpretation for it. However, it should not be difficult to develop a proper economic interpretation of Cauchy's condition $\gamma(\tau)=$ $F(\alpha(\tau), \beta(\tau))$ as the one given below. Hence, if we assume that Cauchy's condition makes economic sense and (III.7) is true, then we have a unique solution of the 'Cauchy problem for EE', and if the spatial curve $\vec{r}=\vec{r}(\tau)=(\alpha(\tau), \beta(\tau), \gamma(\tau))$ is smooth enough, $Y=F(K, L))$ will depend continuously on this data curve. Therefore, we have a well posed problem in the sense of Hadamard. More precisely, if for a given production activity, we have 'data' in the form of the following time series:

$$
\{(K(\tau), L(\tau), Y(\tau))=(\alpha(\tau), \beta(\tau), \gamma(\tau)): T \in[0, T]\}
$$

If $Y=F(K, L)$ is the production function of the given production activity, then $\gamma(\tau)=F(\alpha(\tau), \beta(\tau))$. Moreover, if the Jacobian determinant:

$$
J=\left|\begin{array}{cc}
\alpha^{\prime}\left(\tau_{0}\right) & K\left(\tau_{0}\right) \\
\beta^{\prime}\left(\tau_{0}\right) & L\left(\tau_{0}\right)
\end{array}\right|=\left|\begin{array}{cc}
\alpha^{\prime}\left(\tau_{0}\right) & \alpha\left(\tau_{0}\right) \\
\beta^{\prime}\left(\tau_{0}\right) & \beta\left(\tau_{0}\right)
\end{array}\right| \neq 0
$$

Then, the production function $Y=F(K, L)$ of such activity is uniquely determined in the neighborhood of $\tau_{0}$ by the Cauchy problem (III.3), assuming that the given production activity has CRS. Therefore, to solve this hypothetical ideal case, we do not need any additional conditions to determine the production function $Y=$ $F(K, L)$. However, as previously stated, problem (III.3) is compatible with imposing additional conditions on its solutions (III.6), for example, the law of diminishing marginal returns. We develop this additional restriction on the solution of (III.3) in Section IV. To illustrate the method developed above, we now offer two examples.

(Ex. 1) Consider a productive activity for which we have the time series data $(\alpha(\tau), \beta(\tau), \gamma(\tau))=(\tau, l, \gamma(\tau))$, where $\gamma(\tau)$ is an arbitrary function of $\tau$ and $\tau \in[0 . T]$.

First, it is easy to verify that:

$$
J=\left|\begin{array}{cc}
\alpha^{\prime}(\tau) & \mathrm{T} \\
\beta^{\prime}(\tau) & 1
\end{array}\right|=\left|\begin{array}{ll}
1 & \tau \\
0 & 1
\end{array}\right|=1 \neq 0
$$

Then, $K \frac{\partial F}{\partial K}+L \frac{\partial F}{\partial L}=F$ such that $\gamma(\tau)=F(\tau, 1)$ has a unique solution. Using the previously described methodology, we find that the solution is $Y=F(K, L)=$ $L \gamma\left(\frac{K}{L}\right)$, which is clearly HDO. However, this solution does not necessarily satisfy the properties $\frac{\partial F}{\partial K}>0, \frac{\partial F}{\partial L}>0$, $\frac{\partial^{2} F}{\partial K^{2}}<0$, and $\frac{\partial^{2} F}{\partial L^{2}}<0$ because if $\gamma(\tau)=\sin (\tau)$, then $Y=F(K, L)=L \sin \left(\frac{K}{L}\right), \quad$ and $\quad \frac{\partial F}{\partial K}=\cos \left(\frac{K}{L}\right) ; \frac{\partial F}{\partial L}=$ $\sin \left(\frac{K}{L}\right)-\frac{K}{L} \cos \left(\frac{K}{L}\right) ; \frac{\partial^{2} F}{\partial K^{2}}=-\frac{1}{L} \sin \left(\frac{K}{L}\right)$ and $\frac{\partial^{2} F}{\partial L^{2}}=$ $-\frac{K^{2}}{L^{3}} \sin \left(\frac{K}{L}\right)$, and none of these relations have a determined fixed sign.

(Ex. 2) We assume the same Cauchy's condition as in the previous example. However, we now consider $\gamma(\tau)=A \tau^{m}$, where $A$ is a positive constant and $m \in[0,1]$. Then, $Y=F(K, L)=A L\left(\frac{K}{L}\right)^{m}=A K^{m} L^{1-m}$, which is the Cobb-Douglas function, and it is easy to verify that $\frac{\partial F}{\partial K}=\mathrm{mA}\left(\frac{K}{L}\right)^{m-1}>0 ; \frac{\partial F}{\partial L}=(1-\mathrm{m}) \mathrm{A}\left(\frac{K}{L}\right)^{m}>0 ; \frac{\partial^{2} F}{\partial K^{2}}=$ $\mathrm{m}(\mathrm{m}-1) \mathrm{A}\left(\frac{K}{L}\right)^{m-2}\left(\frac{1}{L}\right)<0$ and $\frac{\partial^{2} F}{\partial L^{2}}=\mathrm{m}(1-$ m)A $\left(\frac{K}{L}\right)^{m}\left(\frac{-1}{L^{2}}\right)<0$. 
Note that the curve $\Gamma: \vec{r}=\vec{r}(\tau)=(\alpha(\tau), \beta(\tau), \gamma(\tau))=(\tau, 1, \gamma(\tau)) \quad$ (on which we imposed Cauchy's condition $\gamma(\tau)=F(\tau, 1)$ in the above two examples) can be interpreted as follows: in the period of time $[0, T]$, we fix the quantity of labor and increase the capital proportionally to time (with a factor of proportionality equal to one), and the production output is $\gamma(\tau)$. Hence, it is sensible to suppose that $\gamma(\tau)$ must be an increasing function of $\tau$ and that the function $\gamma(\tau)=$ $\sin (\tau)$ is not necessarily an increasing function of $\tau$, unless we restrict it to the period of time $\left[0, \frac{\pi}{2}\right]$, where we have $\frac{\partial F}{\partial K}=\cos \left(\frac{K}{L}\right) ; \frac{\partial F}{\partial L}=\sin \left(\frac{K}{L}\right)-\frac{K}{L} \cos \left(\frac{K}{L}\right) ; \frac{\partial^{2} F}{\partial K^{2}}=$ $-\frac{1}{L} \sin \left(\frac{K}{L}\right)$ and $\frac{\partial^{2} F}{\partial L^{2}}=-\frac{K^{2}}{L^{3}} \sin \left(\frac{K}{L}\right)$. Then, if $\frac{K}{L} \epsilon\left[0, \frac{\pi}{2}\right]$, $\frac{\partial F}{\partial K}$ and $\frac{\partial F}{\partial L}$ change signs, $\frac{\partial^{2} F}{\partial K^{2}}$ and $\frac{\partial^{2} F}{\partial L^{2}}$ will always be negative. Therefore, even though we impose a sensible restriction on the function $\gamma(\tau)$ from the economic point of view, we still have a solution that does not satisfy the law of diminishing marginal returns. What other sensible economic restrictions can we impose on the function $\gamma(\tau)$ ? We can ask that it be convex. However, $\gamma(\tau)=\sin (\tau)$ is convex on the interval $\left[0, \frac{\pi}{2}\right]$. Hence, we have a solution to problem (III.3) that does not satisfy the law of diminishing marginal returns under very sensible restrictions on the function $\gamma(\tau)$ and on Cauchy's condition $\gamma(\tau)=F(\tau, 1)$.

At this point, the reader may object that the period of time $\left[0, \frac{\pi}{2}\right]$ is too short or too long and does not make sense from an economic point of view. However, such objections can be easily solved by rescaling the function $\gamma(\tau)$. For example, instead of taking $\gamma(\tau)=\sin (\tau)$, we can take $\gamma(\tau)=\sin \left(\frac{\tau}{\eta}\right)$ with $\eta \in \mathbb{R}^{+}$. Now, the time interval where $\gamma(\tau)$ is increasing and convex is $\left[0, \eta \frac{\pi}{2}\right]$ and $Y=$ $F(K, L)=L \sin \left(\frac{K}{\eta L}\right)$ is the solution to the problem:

$$
\begin{gathered}
K \frac{\partial F}{\partial K}+L \frac{\partial F}{\partial L}=F \text { such that } \sin \left(\frac{\tau}{\eta}\right)=F(\tau, 1) \text { and } \\
Y=F(K, L)
\end{gathered}
$$

Nevertheless, this does not satisfy the law of diminishing marginal returns. However, it is well known that the solution of example (2) satisfies the law of diminishing marginal returns. Hence, for the solution of problem (III.3) to satisfy additional properties or laws, they must be imposed on it. Then, the general solution to the problem is as follows:

$$
\left\{\begin{array}{l}
K=K(\tau, t)=\alpha(\tau) e^{t} \\
L=L(\tau, t)=\beta(\tau) e^{t} \\
Y=Y(\tau, t)=\gamma(\tau) e^{t}
\end{array}\right.
$$

Additional properties or laws will restrict the choices of $\alpha(\tau), \beta(\tau)$ and $\gamma(\tau)$ (as shown in Section IV) without losing the existence and uniqueness of the solution of problem (III.3) if $J \neq 0$. Hence, every solution of problem (III.4) can be adapted to a solution of problem (III.3). However, the inverse is not true, as we have already shown with example (1) above. In the next section, we analyze the solution to problem (III.3) and discover some consequences. In contrast, to obtain additional properties or characteristics of the protoproduction functions, we apply all the advanced calculus for functions of several variables and differential geometry we choose to the surface represented parametrically by (III.6), namely:

$$
\left\{\begin{array}{l}
K=K(\tau, t)=\alpha(\tau) e^{t} \\
L=L(\tau, t)=\beta(\tau) e^{t} \quad \tau \in[0, T] \\
Y=Y(\tau, t)=\gamma(\tau) e^{t}
\end{array}\right.
$$

In particular, we can (a) define isoquants (level curves); (b) obtain the tangent plane (or differential as a linear approximation of the total differential or absolute change of the production function in a neighborhood of the point of operation); (c) obtain the normal vector and normal line to the production function at the point of operation; (d) obtain local coordinate systems on the tangent plane; (e) determine the lengths, angles, and areas on the integral surface or production function; (f) obtain the fundamental quadratic form of the integral surface or production function; (g) obtain the curvature of the integral surface or production function; (h) obtain the second fundamental quadratic form of the integral surface or production function; and (i) obtain the intrinsic properties of the integral surface or production function ${ }^{10}$. Moreover, because the Jacobian matrix (III.9) differs from zero, it follows from the inverse function theorem that the transformation

$$
\left\{\begin{array}{l}
K=K(\tau, t)=\alpha(\tau) e^{t} \\
L=L(\tau, t)=\beta(\tau) e^{t}
\end{array}\right\}
$$

is locally bijective (i.e., it is locally invertible). Therefore, (III.9) guarantees that locally (III.6) represents a surface $\Sigma$ of the form:

$$
Y=F(K, L)
$$

where (III.6) is the parametric representation of the same surface. In other words, (III.6) and (III.4) represent the same unique production function to which we can apply advanced calculus and differential geometry to quantify its properties, where the curve $\Gamma:(\alpha(\tau), \beta(\tau), \gamma(\tau))$ is the curve obtained from the empirical data of the given production activity. 


\section{The Necessary and Sufficient Conditions for the Solution (III.6) to Satisfy the Law of Diminishing Marginal Returns ${ }^{11}$}

Thus far, the production function $Y=F(K, L)$ has been defined parametrically by the following equations:

$$
\left\{\begin{array}{l}
K=K(\tau, t)=\alpha(\tau) e^{t} \\
L=L(\tau, t)=\beta(\tau) e^{t} \quad \tau \in[0, T] \\
Y=Y(\tau, t)=\gamma(\tau) e^{t}
\end{array}\right.
$$

It is known that $Y=F(K, L)$ satisfies the law of diminishing marginal returns if:

$$
\frac{\partial Y}{\partial K}>0, \frac{\partial Y}{\partial L}>0, \frac{\partial^{2} Y}{\partial K^{2}}<0, \text { and } \frac{\partial^{2} Y}{\partial L^{2}}<0
$$

From (III.6), the conditions for $\frac{\partial Y}{\partial K}>0$ are as follows:

$$
\begin{aligned}
1 & =\alpha^{\prime}(\tau) e^{t} \frac{\partial \tau}{\partial K}+\alpha(\tau) e^{t} \frac{\partial t}{\partial K} \\
0 & =\beta^{\prime}(\tau) e^{t} \frac{\partial \tau}{\partial K}+\beta(\tau) e^{t} \frac{\partial t}{\partial K} \\
\frac{\partial t}{\partial K} & =\gamma^{\prime}(\tau) e^{y} \frac{\partial \tau}{\partial K}+\gamma(\tau) e^{t} \frac{\partial t}{\partial K}
\end{aligned}
$$

From the first two equations above, we obtain:

$$
\frac{\partial \tau}{\partial K}=\frac{\left|\begin{array}{ll}
1 & \alpha(\tau) \\
0 & \beta(\tau)
\end{array}\right|}{\left|\begin{array}{ll}
\alpha^{\prime}(\tau) & \alpha(\tau) \\
\beta^{\prime}(\tau) & \beta(\tau)
\end{array}\right|} \frac{e^{t}}{e^{2 t}}=\frac{\beta(\tau) e^{-t}}{\alpha^{\prime}(\tau) \beta(\tau)-\beta^{\prime}(\tau) \alpha(\tau)}
$$

and

$$
\frac{\partial t}{\partial K}=\frac{\left|\begin{array}{ll}
\alpha^{\prime}(\tau) & 1 \\
\beta^{\prime}(\tau) & 0
\end{array}\right|}{\left|\begin{array}{ll}
\alpha^{\prime}(\tau) & \alpha(\tau) \\
\beta^{\prime}(\tau) & \beta(\tau)
\end{array}\right|} \frac{e^{t}}{e^{2 t}}=\frac{-\beta^{\prime}(\tau) e^{-t}}{\alpha^{\prime}(\tau) \beta(\tau)-\beta^{\prime}(\tau) \alpha(\tau)}
$$

Note that the denominator is equal to the previously defined Jacobian matrix (see (III.9)), and therefore, differs from zero. Then, by substituting this result into the third equation of the system above and by assuming that $\alpha(\tau)>0, \beta(\tau)>0$ and $\gamma(\tau)>0$, we obtain:

$$
\begin{gathered}
\frac{\partial Y}{\partial K}=\frac{\gamma^{\prime}(\tau) \beta(\tau)-\gamma(\tau) \beta^{\tau}}{\alpha^{\prime}(\tau) \beta(\tau)-\beta^{\prime}(\tau) \alpha(\tau)} \\
\Rightarrow\left[\begin{array}{lll}
\frac{\partial Y}{\partial K}>0 \Leftrightarrow\left\{\begin{array}{lll}
\left(\gamma^{\prime} \beta-\beta^{\prime} \gamma\right)>0 & \wedge & \left(\alpha^{\prime} \beta-\beta^{\prime} \alpha\right)>0 \\
\left.\gamma^{\prime} \beta-\beta^{\prime} \gamma\right)<0 & \wedge & \wedge
\end{array}\right. \\
\left.\alpha^{\prime} \beta-\beta^{\prime} \alpha\right)<0
\end{array}\right\} \Leftrightarrow \\
\left\{\begin{array}{lll}
\left(\gamma^{\prime} \beta>\beta^{\prime} \gamma\right) & \wedge & \left(\alpha^{\prime} \beta>\beta^{\prime} \alpha\right) \\
\left(\gamma^{\prime} \beta<\beta^{\prime} \gamma\right) & \wedge & \left(\alpha^{\prime} \beta<\beta^{\prime} \alpha\right)
\end{array}\right\} \Leftrightarrow\left\{\begin{array}{lll}
\frac{\gamma^{\prime}}{\gamma}>\frac{\beta^{\prime}}{\beta} & \wedge & \frac{\alpha^{\prime}}{\alpha}>\frac{\beta^{\prime}}{\beta} \\
\frac{\gamma^{\prime}}{\gamma}<\frac{\beta^{\prime}}{\beta} & \text { or } & \frac{\alpha^{\prime}}{\alpha}<\frac{\beta^{\prime}}{\beta}
\end{array}\right\}
\end{gathered}
$$

Analogously, we can easily find that $\frac{\partial \tau}{\partial L}=\frac{-\alpha e^{-t}}{\alpha^{\prime} \beta-\beta^{\prime} \alpha}$, $\frac{\partial t}{\partial L}=\frac{\alpha^{\prime} r^{-t}}{\alpha^{\prime} \beta-\beta^{\prime} \alpha}$ and $\frac{\partial Y}{\partial L}=\frac{\left(\alpha^{\prime} \gamma-\gamma^{\prime} \alpha\right)}{\left(\alpha^{\prime} \beta-\beta^{\prime} \alpha\right)}$

Then, $\frac{\partial Y}{\partial L}>0$ if:

$$
\begin{aligned}
\left\{\left(\alpha^{\prime} \gamma-\gamma^{\prime} \alpha\right)>0\right. & \left.\wedge\left(\alpha^{\prime} \beta-\beta^{\prime} \alpha\right)>0\right\} \text { or }\left\{\left(\alpha^{\prime} \gamma-\gamma^{\prime} \alpha\right)<\right. \\
& \left.0 \wedge\left(\alpha^{\prime} \beta-\beta^{\prime} \alpha\right)<0\right\}
\end{aligned}
$$

which is equivalent to:

$$
\left\{\frac{\alpha^{\prime}}{\alpha}>\frac{\gamma^{\prime}}{\gamma} \wedge \frac{\alpha^{\prime}}{\alpha}>\frac{\beta^{\prime}}{\beta}\right\} \text { or }\left\{\frac{\alpha^{\prime}}{\alpha}<\frac{\gamma^{\prime}}{\gamma} \wedge \frac{\alpha^{\prime}}{\alpha}<\frac{\beta^{\prime}}{\beta}\right\}
$$

Therefore, to simultaneously obtain $\frac{\partial Y}{\partial K}>0$ and $\frac{\partial Y}{\partial L}>0$, we have two cases with the following requirements ${ }^{12}$ :

Case I: $\left(\alpha^{\prime} \beta-\beta^{\prime} \alpha\right)>0 ;\left(\alpha^{\prime} \gamma-\gamma^{\prime} \alpha\right)>0 ;\left(\gamma^{\prime} \beta-\right.$ $\left.\beta^{\prime} \gamma\right)>0$. In other words, $\frac{\alpha^{\prime}}{\alpha}>\frac{\gamma^{\prime}}{\gamma}>\frac{\beta^{\prime}}{\beta} ; \alpha(\tau)>0$, $\beta(\tau)>0$ and $\gamma(\tau)>0$, or

Case II: $\left(\alpha^{\prime} \beta-\beta^{\prime} \alpha\right)<0 ;\left(\alpha^{\prime} \gamma-\gamma^{\prime} \alpha\right)<0 ; \quad\left(\gamma^{\prime} \beta-\right.$ $\left.\beta^{\prime} \gamma\right)<0$. In other words, $\frac{\alpha^{\prime}}{\alpha}<\frac{\gamma^{\prime}}{\gamma}<\frac{\beta^{\prime}}{\beta} ; \alpha(\tau)>0$, $\beta(\tau)>0$ and $\gamma(\tau)>0$.

Focusing now on the second derivatives, let us obtain the conditions for $\frac{\partial^{2} Y}{\partial K^{2}}<0$. Because $\frac{\partial Y}{\partial K}=\frac{\gamma^{\prime} \beta-\gamma \beta^{\prime}}{\alpha^{\prime} \beta-\beta^{\prime} \alpha}$ and $\frac{\partial \tau}{\partial K}=\frac{\beta(\tau) e^{-t}}{\alpha^{\prime} \beta-\beta^{\prime} \alpha}$, it is easy to verify that

$$
\begin{aligned}
\frac{\partial^{2} Y}{\partial K^{2}} & =\left[\frac{\left(\gamma^{\prime \prime} \beta-\beta^{\prime \prime} \gamma\right)\left(\alpha^{\prime} \beta-\beta^{\prime} \alpha\right)-\left(\alpha^{\prime \prime} \beta-\beta^{\prime \prime} \alpha\right)\left(\gamma^{\prime} \beta-\beta^{\prime} \gamma\right)}{\left(\alpha^{\prime} \beta-\beta^{\prime} \alpha\right)^{2}}\right] \frac{\partial \tau}{\partial K} \\
& =\left[\frac{\left(\gamma^{\prime \prime} \beta-\beta^{\prime \prime} \gamma\right)\left(\alpha^{\prime} \beta-\beta^{\prime} \alpha\right)-\left(\alpha^{\prime \prime} \beta-\beta^{\prime \prime} \alpha\right)\left(\gamma^{\prime} \beta-\beta^{\prime} \gamma\right)}{\left(\alpha^{\prime} \beta-\beta^{\prime} \alpha\right)^{2}}\right]\left(\frac{\beta(\tau) e^{-\tau}}{\alpha^{\prime} \beta-\beta^{\prime} \alpha}\right)
\end{aligned}
$$

12 The other two combinations of the obtained conditions for $\frac{\partial Y}{\partial K}>0$ and $\frac{\partial Y}{\partial L}>0$ lead to a contradiction. In addition, notice that both partial derivatives are independent of $t$.
11 In this study, we restrict ourselves only to the possibility of diminishing returns so as not to overload the exposure. However, the theory developed here also allows the generation of production functions with constant or increasing marginal returns. 
Because $\beta(\tau) e^{-\tau}>0$, it follows that $\frac{\partial^{2} Y}{\partial K^{2}}>0$ if:

$$
\left\{\begin{array}{ccc}
\left(\gamma^{\prime \prime} \beta-\beta^{\prime \prime} \gamma\right)\left(\alpha^{\prime} \beta-\beta^{\prime} \alpha\right)<\left(\alpha^{\prime \prime} \beta-\beta^{\prime \prime} \alpha\right)\left(\gamma^{\prime} \beta-\beta^{\prime} \gamma\right) & \wedge & \left(\alpha^{\prime} \beta-\beta^{\prime} \alpha\right)>0 \\
\text { or } & & \\
\left(\gamma^{\prime \prime} \beta-\beta^{\prime \prime} \gamma\right)\left(\alpha^{\prime} \beta-\beta^{\prime} \alpha\right)>\left(\alpha^{\prime \prime} \beta-\beta^{\prime \prime} \alpha\right)\left(\gamma^{\prime} \beta-\beta^{\prime} \gamma\right) & \wedge & \left(\alpha^{\prime} \beta-\beta^{\prime} \alpha\right)<0
\end{array}\right\}
$$

Analogously, from $\frac{\partial Y}{\partial L}=\frac{\alpha^{\prime} \gamma-\alpha \gamma^{\prime}}{\left(\alpha^{\prime} \beta-\beta^{\prime} \alpha\right)}$ and $\frac{\partial \tau}{\partial L}=\frac{-\alpha e^{-t}}{\alpha^{\prime} \beta-\beta^{\prime} \alpha}$ we obtain:

$$
\frac{\partial^{2} Y}{\partial L^{2}}=\left[\frac{\left(\alpha^{\prime \prime} \gamma-\gamma^{\prime \prime} \alpha\right)\left(\alpha^{\prime} \beta-\beta^{\prime} \alpha\right)-\left(\alpha^{\prime \prime} \beta-\beta^{\prime \prime} \alpha\right)\left(\alpha^{\prime} \gamma-\gamma^{\prime} \alpha\right)}{\left(\alpha^{\prime} \beta-\beta^{\prime} \alpha\right)^{2}}\right]\left(\frac{-\alpha e^{-t}}{\alpha^{\prime} \beta-\beta^{\prime} \alpha}\right)
$$

Because $\alpha(\tau) e^{-t}>0 ; \frac{\partial^{2} Y}{\partial L^{2}}<0$ if:

$$
\left\{\begin{array}{ccc}
\left(\alpha^{\prime \prime} \gamma-\gamma^{\prime \prime} \alpha\right)\left(\alpha^{\prime} \beta-\beta^{\prime} \alpha\right)>\left(\alpha^{\prime \prime} \beta-\beta^{\prime \prime} \alpha\right)\left(\alpha^{\prime} \gamma-\gamma^{\prime} \alpha\right) & \wedge & \left(\alpha^{\prime} \beta-\beta^{\prime} \alpha\right)>0 \\
\text { or } & & \\
\left(\alpha^{\prime \prime} \gamma-\gamma^{\prime \prime} \alpha\right)\left(\alpha^{\prime} \beta-\beta^{\prime} \alpha\right)<\left(\alpha^{\prime \prime} \beta-\beta^{\prime \prime} \alpha\right)\left(\alpha^{\prime} \gamma-\gamma^{\prime} \alpha\right) & \wedge & -\left(\alpha^{\prime} \beta-\beta^{\prime} \alpha\right)<0
\end{array}\right\}
$$

Note that (V.2) is compatible with (V.4) and (V.3) is compatible with (V.5). The other two combinations are contradictory.

Then, from conditions (V.2) and (V.4), we obtain $\frac{\partial^{2} Y}{\partial K^{2}}<0$ and $\frac{\partial^{2} Y}{\partial L^{2}}<0$. It follows that:

$$
\begin{gathered}
\left\{\begin{array}{c}
\left(\gamma^{\prime \prime} \beta-\beta^{\prime \prime} \gamma\right)\left(\alpha^{\prime} \beta-\beta^{\prime} \alpha\right)<\left(\alpha^{\prime \prime} \beta-\beta^{\prime \prime} \alpha\right)\left(\gamma^{\prime} \beta-\beta^{\prime} \gamma\right) \wedge\left(\alpha^{\prime} \beta-\beta^{\prime} \alpha\right)>0 \\
\text { or } \\
\left(\gamma^{\prime \prime} \beta-\beta^{\prime \prime} \gamma\right)\left(\alpha^{\prime} \beta-\beta^{\prime} \alpha\right)>\left(\alpha^{\prime \prime} \beta-\beta^{\prime \prime} \alpha\right)\left(\gamma^{\prime} \beta-\beta^{\prime} \gamma\right) \wedge\left(\alpha^{\prime} \beta-\beta^{\prime} \alpha\right)<0
\end{array}\right\} \\
\frac{\left(\gamma^{\prime \prime} \beta-\beta^{\prime \prime} \gamma\right)}{\left(\gamma^{\prime} \beta-\beta^{\prime} \gamma\right)}<\frac{\left(\alpha^{\prime \prime} \beta-\beta^{\prime \prime} \alpha\right)}{\left(\alpha^{\prime} \beta-\beta^{\prime} \alpha\right)}<\frac{\left(\alpha^{\prime \prime} \gamma-\gamma^{\prime \prime} \alpha\right)}{\left(\alpha^{\prime} \gamma-\gamma^{\prime} \alpha\right)}
\end{gathered}
$$

Assuming that $\left(\alpha^{\prime} \gamma-\gamma^{\prime} \alpha\right)>0 ;\left(\alpha^{\prime} \gamma-\gamma^{\prime} \alpha\right)>0$ and $\left(\gamma^{\prime} \beta-\beta^{\prime} \gamma\right)>0$, and from conditions (V.3) and (V.5), $\frac{\partial^{2} Y}{\partial K^{2}}<0$ and $\frac{\partial^{2} Y}{\partial L^{2}}<0$, it follows that:

$$
\frac{\left(\gamma^{\prime \prime} \beta-\beta^{\prime \prime} \gamma\right)}{\left(\gamma^{\prime} \beta-\beta^{\prime} \gamma\right)}>\frac{\left(\alpha^{\prime \prime} \beta-\beta^{\prime \prime} \alpha\right)}{\left(\alpha^{\prime} \beta-\beta^{\prime} \alpha\right)}>\frac{\left(\alpha^{\prime \prime} \gamma-\gamma^{\prime \prime} \alpha\right)}{\left(\alpha^{\prime} \gamma-\gamma^{\prime} \alpha\right)}
$$

assuming that $\left(\alpha^{\prime} \beta-\beta^{\prime} \alpha\right)<0 ;\left(\alpha^{\prime} \gamma-\gamma^{\prime} \alpha\right)<0$ and $\left(\gamma^{\prime} \beta-\beta^{\prime} \gamma\right)<0$.

Note that (IV.2) and (IV.4) are compatible with Case 1 and contradict Case II. Analogously, (IV.3) and (IV.5) are compatible with Case II and contradict case I. We summarize these results in the following two propositions:

Proposition 1: (Necessary and sufficient conditions) $0<\frac{\partial Y}{\partial K} ; 0<\frac{\partial Y}{\partial L} ; \frac{\partial^{2} Y}{\partial K^{2}}<0$ and $\frac{\partial^{2} Y}{\partial L^{2}}<0$ if and only if: (1.1) $0<\alpha(\tau) ; 0<\beta(\tau)$; and $0<\gamma(\tau), \forall \tau \in[0, T]$ (1.2) $\frac{\beta^{\prime}}{\beta}<\frac{\gamma^{\prime}}{\gamma}<\frac{\alpha^{\prime}}{\alpha} ; \forall \tau \in[0, T]$

(1.3) $\left(\frac{\gamma^{\prime \prime} \beta-\beta^{\prime \prime} \gamma}{\gamma^{\prime} \beta-\beta^{\prime} \gamma}\right)<\left(\frac{\alpha^{\prime \prime} \beta-\beta^{\prime \prime} \alpha}{\alpha^{\prime} \beta-\beta^{\prime} \alpha}\right)<\left(\frac{\alpha^{\prime \prime} \gamma-\gamma^{\prime \prime} \alpha}{\alpha^{\prime} \gamma-\gamma^{\prime} \alpha}\right) ; \forall \tau \in$ $[0, T]$

Proposition 2: (Necessary and sufficient conditions) $0<\frac{\partial Y}{\partial K} ; 0<\frac{\partial Y}{\partial L} ; \frac{\partial^{2} Y}{\partial K^{2}}<0$ and $\frac{\partial^{2} Y}{\partial L^{2}}<0$ if and only if:

(2.1) $0<\alpha(\tau) ; 0<\beta(\tau)$; and $0<\gamma(\tau), \forall \tau \in[0, T]$

(2.2) $\frac{\alpha^{\prime}}{\alpha}<\frac{\gamma^{\prime}}{\gamma}<\frac{\beta^{\prime}}{\beta} ; \forall \tau \in[0, T]$

(2.3) $\left(\frac{\alpha^{\prime \prime} \gamma-\gamma^{\prime \prime} \alpha}{\alpha^{\prime} \gamma-\gamma^{\prime} \alpha}\right)<\left(\frac{\alpha^{\prime \prime} \beta-\beta^{\prime \prime} \alpha}{\alpha^{\prime} \beta-\beta^{\prime} \alpha}\right)<\left(\frac{\gamma^{\prime \prime} \beta-\beta^{\prime \prime} \gamma}{\gamma^{\prime} \beta-\beta^{\prime} \gamma}\right) ; \forall \tau \in[0, T]$

Note that inequalities (1.2), (1.3), (2.2), and (2.3) can be integrated between 0 and $\tau \in[0, T]$, from which we obtain the following inequalities:

$(1.2) \frac{\beta(\tau)}{\beta(0)}<\frac{\gamma(\tau)}{\gamma(0)}<\frac{\alpha(\tau)}{\alpha(0)} ; \forall \tau \in[0, T]$

(1.3) $\left(\frac{\gamma^{\prime}(\tau) \beta(\tau)-\beta^{\prime}(\tau) \gamma(\tau)}{\gamma^{\prime}(0) \beta(0)-\beta^{\prime}(0) \gamma(0)}\right)<\left(\frac{\alpha^{\prime}(\tau) \beta(\tau)-\beta^{\prime}(\tau) \alpha(\tau)}{\alpha^{\prime}(0) \beta(0)-\beta^{\prime}(0) \alpha(0)}\right)<$

$\left(\frac{\alpha^{\prime}(\tau) \gamma(\tau)-\gamma^{\prime}(\tau) \alpha(\tau)}{\alpha^{\prime}(0) \gamma(0)-\gamma^{\prime}(0) \alpha(0)}\right) ; \forall \tau \in[0, T]$

(2.2) $\frac{\alpha(\tau)}{\alpha(0)}<\frac{\gamma(\tau)}{\gamma(0)}<\frac{\beta(\tau)}{\beta(0)} ; \forall \tau \in[0, T]$

(1.2) $\left(\frac{\alpha^{\prime}(\tau) \gamma(\tau)-\gamma^{\prime}(\tau) \alpha(\tau)}{\alpha^{\prime}(0) \gamma(0)-\gamma^{\prime}(0) \alpha(0)}\right)<\left(\frac{\alpha^{\prime}(\tau) \beta(\tau)-\beta^{\prime}(\tau) \alpha(\tau)}{\alpha^{\prime}(0) \beta(0)-\beta^{\prime}(0) \alpha(0)}\right)<$ $\left(\frac{\gamma^{\prime}(\tau) \beta(\tau)-\beta^{\prime}(\tau) \gamma(\tau)}{\gamma^{\prime}(0) \beta(0)-\beta^{\prime}(0) \gamma(0)}\right) ; \forall \tau \in[0, T]$ 
Thus far, we have focused our attention on the solution to problem (III.3) from an aggregate point of view. In the next section, we study the relation of self-similar solutions to problem (III.3) and the Inada conditions for the corresponding per capita production function (i.e., $y=F(k)=F\left(\frac{K}{L}\right)$, with $\left.y=\frac{Y}{L}\right)$ because the Inada conditions are additional restrictions that standard production functions have to meet to define a per capita production function.

\section{Self-Similar Solutions to Problem (III.3) and the Inada Conditions}

In this section, we define a self-similar partial differential equation (PDE) solution, obtaining an ordinary differential equation (ODE) from the PDE by reducing the number of independent variables to only one through an appropriate change of variables. Then, given the problem (III.3) posed before:

$\left\{\begin{array}{ccc}K \frac{\partial F}{\partial K}+L \frac{\partial F}{\partial L} & = & F, \text { with } Y=F(K, L) \text { and si } \\ \gamma(\tau) & = & F(\alpha(\tau), \beta(\tau)), \text { when } \tau \in\end{array}\right.$

Let us search for solutions to this problem in the following form:

$$
Y=F(K, L)=L^{\mu} F(\varsigma) \text { such that } \varsigma=\frac{K}{L}
$$

where $\mu$ is a priori positive number. From (V.1), it is easy to verify that

$$
\begin{array}{ll}
\frac{\partial F}{\partial K} & =L^{\mu} F^{\prime}(\varsigma) \frac{\partial \varsigma}{\partial K}=L^{\mu} F^{\prime}(\varsigma)\left(\frac{1}{L}\right)=L^{\mu-1} F^{\prime}(\varsigma) \\
\frac{\partial F}{\partial L} & =\mu L^{\mu-1} F^{\prime}(\varsigma)+L^{\mu} F^{\prime}(\varsigma) \frac{\partial \varsigma}{\partial L}=\mu L^{\mu-1} F^{\prime}(\varsigma)+L^{\mu} F^{\prime}(\varsigma)\left(-\frac{K}{L^{2}}\right) \\
\Rightarrow K \frac{\partial F}{\partial K}+L \frac{\partial F}{\partial L} & =\mu L^{\mu-1} F^{\prime}(\varsigma)-K L^{\mu-2} F^{\prime}(\varsigma) \\
& =K L^{\mu-1} F^{\prime}(\varsigma)+\mu L^{\mu} F(\varsigma)-K t^{\mu-1} F^{\prime}(\varsigma) \\
& =\mu L^{\mu} F(\varsigma) \\
\Rightarrow \quad & \left\{\mu L^{\mu} F(\varsigma)=L^{\mu} F(\varsigma) \Leftrightarrow \mu=1\right\}
\end{array}
$$

Therefore, the only positive value ensuring that (II.3) has a solution of the form (V.1) is $\mu=1$. Note that for the function $Y=F(K, L)=L F(\varsigma)$, where $\varsigma=\left(\frac{K}{L}\right)$ ) and $F(\cdot)$, any given function is not only a solution of Euler's PDE: $K \frac{\partial F}{\partial K}+L \frac{\partial F}{\partial L}=F$ but also reduces $\mathrm{EE}$ to an identity because $\quad \frac{\partial Y}{\partial K}=F^{\prime}(\varsigma) \quad, \quad \frac{\partial Y}{\partial L}=F(\varsigma)-\frac{K}{L} F^{\prime}(\varsigma) \Rightarrow$ $K \frac{\partial Y}{\partial K}+\mathrm{L} \frac{\partial Y}{\partial L}=L F(\varsigma)=Y$. Now, from $Y=L F\left(\frac{K}{L}\right)$, the following definition can be made: $Y=F(k)$ if we define $y=\frac{Y}{L}$ (output per capita) and $k=\frac{K}{L}$ (capital per capita).
What type of curves are $y=F(k)$ ? What is the relation of this type of curve to the integral surface $Y=F(K, L)$ and its characteristic curves? If $\varsigma=\varsigma_{0}=$ constant, i.e., the value of $\varsigma \Rightarrow \frac{K}{L}=\varsigma_{0} \Rightarrow K=\varsigma_{0} L \Rightarrow$ on a characteristic projection of one of the 'characteristic curves' that form the 'integral surface' or production function: $Y=F(K, L)$. If we remember that $\mathrm{EE}$ is singular at the origin and all its characteristic projections spring from the origin, the consequence of these observations is that we cannot take the initial condition at $(K, L)=(0,0)$. In fact, if we do so, $\varsigma=\frac{K}{L}$ is undefined. In particular, we can never take the initial condition of the form $(\mathrm{K}, 0)$. By symmetry, we can look for a solution of $\mathrm{EE}$ in the following form:

$$
Y=F(K, L)=L^{v} F(\sigma) \text { such that } \sigma=\frac{L}{K^{\prime}}
$$

which yields $Y=K F\left(\frac{L}{K}\right)$, which may or may not make economic sense. Hence, it is better that we do not take initial conditions of the form $(0, \mathrm{~L})$ either. Therefore, we take the domain $\mathrm{N} \subseteq \mathbb{R}^{+} \times \mathbb{R}^{+}$in (III.1). Conversely, the solution of $\mathrm{EE}$ of the form $Y=L F(K)$ can also be obtained directly from the CRS property because EE and this property are equivalent. In fact, if $F(\lambda K, \lambda L)=\lambda Y \Rightarrow$ $\left\{\right.$ if $\left.\lambda=L \Rightarrow F\left(\frac{K}{L}, 1\right)=\frac{Y}{L}\right\} \Rightarrow Y=L F\left(\frac{K}{L}\right)$, then analogously, we can show that taking $\lambda=\frac{1}{K}, F\left(1, \frac{L}{K}\right)=\frac{Y}{K} \Leftrightarrow Y=$ $K F\left(\frac{L}{L}\right)$.

Proof: Consider EE, $K \frac{\partial F}{\partial K}+L \frac{\partial F}{\partial L}=F$, and introduce the change of variables:

$$
\begin{aligned}
& \varsigma=\varsigma(K, L)=\frac{K}{L} \\
& v=v(K, L)=L
\end{aligned}
$$

Note that $\left|\frac{\partial(s, v)}{\partial(K, L)}\right|=\left|\begin{array}{cc}\frac{1}{L} & -\frac{K}{L^{2}} \\ 0 & 1\end{array}\right|=\frac{1}{L} \neq 0$ and, therefore, there is an invertible change of variables.

Conversely:

$$
\begin{array}{ll}
\frac{\partial F}{\partial K} & =\frac{\partial F}{\partial \varsigma} \frac{\partial \varsigma}{\partial K}+\frac{\partial F}{\partial v} \frac{\partial v}{\partial K}=\frac{1}{L} \frac{\partial F}{\partial \varsigma} \\
\frac{\partial F}{\partial L} & =\frac{\partial F}{\partial \varsigma} \frac{\partial \varsigma}{\partial L}+\frac{\partial F}{\partial v} \frac{\partial v}{\partial L}=\left(-\frac{K}{L^{2}}\right) \frac{\partial F}{\partial \varsigma}+\frac{\partial F}{\partial v} \\
\Rightarrow K \frac{\partial F}{\partial K}+L \frac{\partial F}{\partial L} & =K \frac{\partial F}{\partial K}+L \frac{\partial F}{\partial L}=\frac{K}{L} \frac{\partial F}{\partial \varsigma}-\frac{K}{L} \frac{\partial F}{\partial \varsigma}+L \frac{\partial F}{\partial v}=L \frac{\partial F}{\partial v} \\
\Rightarrow E E \text { for } Y & =F(\varsigma, v) \text { reduces to } \\
& v \frac{\partial F}{\partial v}=F \Leftrightarrow v \frac{\partial Y}{\partial v}=Y \text { : since } v=L
\end{array}
$$

Now, we introduce the transformation:

$$
Y=v y \Rightarrow \frac{\partial Y}{\partial v}=y+v \frac{\partial y}{\partial v}
$$


Then,

$$
\begin{aligned}
& v \frac{\partial y}{\partial v}=v\left(y+v \frac{\partial y}{\partial v}\right)=\text { vy if } \frac{\partial y}{\partial v}=0 \\
& \Rightarrow \frac{\partial y}{\partial v}=0(\text { because } v \neq 0) \Rightarrow y=y(\varsigma)=y\left(\left(\frac{K}{L}\right)\right) \\
& \Rightarrow Y=F(K, L)=L y\left(\frac{K}{L}\right)
\end{aligned}
$$

which is consistent with the previous calculations.

The last calculation proves that $y=\left(\frac{Y}{L}\right)=F\left(\frac{K}{L}\right)=$ $F(\varsigma)$ does not depend on $v=L$; it is only a function of $\varsigma=\left(\frac{K}{L}\right)$, using $\varsigma=\varsigma(K, L)=\frac{K}{L}$ and $v=v(K, L)=L$ as independent variables. Therefore, using the initial condition $y_{0}=y\left(\varsigma_{0}\right)$, where $\varsigma_{0}=\frac{K}{L} \neq 0 \Rightarrow$, the function $y=y(\varsigma)$ is a one-dimensional curve defined by the projection of the characteristic curve of EE, which is $L=\left(\frac{1}{\varsigma_{0}}\right) K$ (i.e., on the straight line that springs from the origin with a slope equal to $\left.\left(\frac{1}{s_{0}}\right)\right)$. In other words, the function $y=y(\varsigma)$ is a one-dimensional curve defined on a straight line where $K$ is proportional to $L$ with a factor of proportionality equal to $\varsigma_{0}$ (i.e., $K=\varsigma_{0} L$, with $\varsigma_{0} \neq 0$ ). In contrast, because $Y=F(K, L)=L y\left(\frac{K}{L}\right), Y$ is a function whose domain of variation is exactly the same where $y=y(\varsigma)$ varies, namely, $K=\varsigma_{0} L$. However, it is modulated by the variable $v=L$ along the straight line $K=\varsigma_{0} L$. In addition, because $Y=F(K, L)=L F\left(\frac{K}{L}\right)$ is a solution of EE and $y=F\left(\frac{K}{L}\right)$ is not a solution of EE, it follows that $Y=F(K, L)=L F\left(\frac{K}{L}\right)=L y\left(\frac{K}{L}\right) \quad$ is a characteristic curve of the integral surface $\mathrm{Y}=F(K, L)$, but $y=y\left(\frac{K}{L}\right)=F\left(\frac{K}{L}\right)$ is not a characteristic curve of $Y=F(K, L)$, even though both are defined on the same characteristic projection of the characteristic curve defined by $K=\varsigma_{0} L$.

Check: $Y=L F\left(\frac{K}{L}\right)$ is an EE solution:

$$
\begin{aligned}
\frac{\partial Y}{\partial K} & =L F^{\prime}\left(\frac{K}{L}\right)\left(\frac{1}{L}\right)=F^{\prime}\left(\frac{K}{L}\right) \\
\frac{\partial Y}{\partial L} & =K\left(\frac{K}{L}\right)+L F^{\prime}\left(\frac{K}{L}\right)\left(-\frac{K}{L^{2}}\right) \\
\Rightarrow K \frac{\partial Y}{\partial K}+L \frac{\partial Y}{\partial L} & =K F^{\prime}\left(\frac{K}{L}\right)+L F\left(\frac{K}{L}\right)-K F^{\prime}\left(\frac{K}{L}\right) \\
& =L F\left(\frac{K}{L}\right)=Y
\end{aligned}
$$

Check: $y=F\left(\frac{K}{L}\right)$ is not an EE solution:

$$
\begin{array}{ll}
\frac{\partial y}{\partial K} & =F^{\prime}\left(\frac{K}{L}\right)\left(\frac{1}{L}\right) \\
\frac{\partial y}{\partial L} & =F^{\prime}\left(\frac{K}{L}\right)\left(-\frac{K}{L^{2}}\right) \\
\Rightarrow K \frac{\partial y}{\partial K}+L \frac{\partial y}{\partial L} & =\left(\frac{K}{L}\right) F^{\prime}\left(\frac{K}{L}\right)-\left(\frac{K}{L}\right) F^{\prime}\left(\frac{K}{L}\right)
\end{array}
$$

These observations answer the questions put forward previously.

Moreover, it is clear that the function $y=y(k)$ can be homogeneous to any degree or not homogeneous with respect to $\mathrm{k}$, which is not a characteristic curve of the production function but a characteristic curve of the production function divided by L. Considering this information, we are now ready to comment on the very well-known Inada conditions. The Inada conditions are additional restrictions imposed on the function $y=$ $f(k)=F(K / L)$, which are $f(0)=0 ; f(\infty)=\infty ; f^{\prime}(k)>$ $0 ; f^{\prime \prime}(k)<0$; $\lim _{k \rightarrow 0} f^{\prime}(k)=\infty$; and $\lim _{k \rightarrow \infty} f^{\prime}(k)=0$. Note that some functions, for example, $y=y(k)=$ $f(k)=A k^{m}$ with $0<m<1$ (which can be obtained from the Cobb-Douglas function), satisfy the Inada Conditions $\quad f(0)=0 ; f(\infty)=\infty ; f^{\prime}(k)=m A k^{m-1}>$ $0 ; f^{\prime \prime}(k)=m(m-1) A k^{m-2}<0 ; \lim _{k \rightarrow 0} f^{\prime}(k)=$ $\lim _{k \rightarrow 0} m A k^{m-1}=\infty$; and $\lim _{k \rightarrow \infty} f^{\prime}(k)=\lim _{k \rightarrow \infty} \frac{m A}{k^{1-m}}=0$. However, let us assume that it is homogeneous of a 'fractional' order equal to $\mathrm{m}$. Hence, it is the type of function discussed previously. In addition to the CobbDouglas production function, there are other production functions that deserve attention. For example:

(a) $y=y(k)=f(k)=A\left\{\frac{k}{1+k}\right\}$ (which can be obtained from the function $Y=F(K, L)=A\left\{\frac{K L}{K+L}\right\}$ that satisfies EE). This function is not homogeneous to any degree and does not satisfy the Inada conditions. In fact:

$f(0)=0 ;$ but $\lim _{k \rightarrow \infty} f(k)=A \neq \infty$;

$f^{\prime}(k)=\frac{A}{(k+1)^{2}}>0$ and $f^{\prime \prime}(k)=-2 A(k+1)^{3}<0 ;$ but

$\lim _{k \rightarrow 0} f^{\prime}(k)=A \neq \infty$ and $\lim _{k \rightarrow \infty} f^{\prime}(k)=0$

(b) $y=y(k)=f(k)=A\left\{\alpha k^{r}+\beta\right\}^{1 / r}$ (which can be obtained from the CES function: $Y=F(K, L)=$ $\left.A\left\{\alpha K^{r}+\beta L^{r}\right\}^{1 / r}\right)$. This function does not satisfy the Inada conditions and is not homogeneous to any degree:

$$
\begin{aligned}
& f(0)=A\{B\}^{1 / r} \neq 0 ; f(\infty)=\infty ; f^{\prime}(k)=\frac{A \alpha k^{r-1}}{\left\{\alpha k^{r}+\beta\right\}^{1-1 / r}}>0 ; \\
& f^{\prime \prime}(k)=\frac{A \alpha(r-1) k^{(r-2)}\left\{\alpha k^{r}+\beta\right\}^{1-1 / r}-A \alpha k^{(r-1)}\left\{\alpha k^{r}+\beta\right\}^{1-1 / r}\left(\alpha r k^{(r-1)}\right)}{\left\{\alpha k^{r}+\beta\right\}^{2(1-1 / r)}} \\
& \frac{A \alpha(r-1) k^{(r-2)}\left\{\alpha k^{r}+\beta\right\}-A r \alpha^{2} k^{2(r-1)}}{\left\{\alpha k^{r}+\beta\right\}^{(2-1 / r)}}<0 ; \\
& \lim _{k \rightarrow 0} f^{\prime}(k)=\infty \text { and } \lim _{k \rightarrow \infty} f^{\prime}(k)=0
\end{aligned}
$$

Although the CES function is a solution of EE, it appears that the Inada conditions are restrictions created to understand the Cobb-Douglas function.

(c) However, there are other solutions to EE that lead to a function $y=f(k)$ that satisfies the Inada conditions. For example:

$$
Y=F(K, L)=\left\{\begin{array}{cc}
0 & , \text { if } K<L \\
A \sqrt{K L}\left(\frac{K^{2}-L^{2}}{K^{2}+L^{2}}\right) & , \text { if } K \geq L
\end{array}\right.
$$


Notice that $Y=F(K, L)$ is HDO. It is a continuous function $\forall(K . L) \in \mathbb{R}^{+} \times \mathbb{R}^{+}$because $Y=F(K, L)=0$ is on line $K=L$. However:

$$
\begin{aligned}
& \frac{\partial y}{\partial K}=\frac{A L}{2 \sqrt{K L}}\left(\frac{K^{2}-L^{2}}{K^{2}+L^{2}}\right)+A \sqrt{K L}\left\{\frac{2 K\left(K^{2}+L^{2}\right)-2 K\left(K^{2}-L^{2}\right)}{\left(K^{2}-L^{2}\right)^{2}}\right\} \\
& =\frac{A}{2} \sqrt{\frac{L}{K}\left(\frac{K^{2}-L^{2}}{K^{2}+L^{2}}\right)}+A \sqrt{K L}\left\{\frac{4 K L^{2}}{\left(K^{2}-L^{2}\right)^{2}}\right\} \\
& \Rightarrow \lim _{K \rightarrow L^{+}} \frac{\partial Y}{\partial K}=A L\left\{\frac{4 L^{3}}{4 L^{4}}\right\}=A\left\{\frac{4 L^{4}}{4 L^{4}}\right\}=A \neq 0 \text {, and } \lim _{K \rightarrow L^{-}} \frac{\partial Y}{\partial K}=0
\end{aligned}
$$

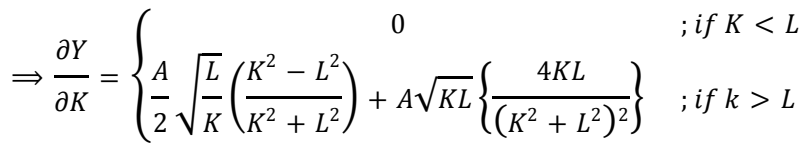

is discontinuous at $K=L$. Analogously, we can show that $\frac{\partial y}{\partial L}$ is discontinuous at $K=L$.

Hence, $Y=F(K, L)$ satisfies EE $\forall(K, L) \in \mathbb{R}^{2}$ such that $\left\{(K, L) \in \mathbb{R}^{2}: K=L\right\}$. From these functions we obtain:

$$
Y=f(k)= \begin{cases}0 & ; \text { if } 0 \leq k<1 \\ A \sqrt{k}\left(\frac{k^{2}-1}{k^{2}+1}\right) & ; \text { if } k \geq 1\end{cases}
$$

These functions are continuous with $\forall k \in[0, \infty]$, $f(0)=0$ and $f(\infty)=\infty$.

Moreover, because

$f^{\prime}(k)= \begin{cases}0 & ; \text { if } 0<k<1 \\ A\left\{\frac{k^{4}+8 k^{2}-1}{2 k^{1 / 2} 2\left(k^{2}+1\right)^{2}}\right\} & ; \text { if } k>1\end{cases}$

It follows that $\lim _{k \rightarrow 1^{-}} f^{\prime}(k)=0$ and $\lim _{k \rightarrow 1^{+}} f^{\prime}(k)=$ $A \neq 0$; therefore, $f^{\prime}(k)$ is discontinuous at $k=1$.

However, $\lim _{k \rightarrow 0} f^{\prime}(k)=\infty$ and $\lim _{k \rightarrow \infty} f^{\prime}(k)=0$.

Additionally, $f^{\prime \prime}(k)=0$ if $k<1$ and $f^{\prime \prime}(k)=$ $A\left(\frac{-k^{6}-33 k^{4}+33 k^{2}+1}{4 k^{3 / 2}\left(k^{2}+1\right)^{3}}\right)$ if $k>1 \Rightarrow \lim _{k \rightarrow 1^{-}} f^{\prime \prime}(k)=0$ and $\lim _{k \rightarrow 1^{+}} f^{\prime \prime}(k)=0$, and therefore, $f^{\prime \prime}(k)$ is continuous at $k=1$ and is equal to zero at $k=1$ (i.e., $f^{\prime \prime}(1)=0$ ). Then, $f^{\prime \prime}(k) \leq 0, \forall k \in[0, \infty]$. Moreover, $y=f(k)$ satisfies all the Inada conditions ${ }^{13}$.

Hence, there are many functions of the form $y=f(k)$ that are homogeneous to a fractional degree, not homogeneous at all, HDO similar to $\mathrm{y}(\mathrm{k})=\mathrm{k}$, and homogeneous to zero degree, or similar to $y(k)=$ Const . However, the function obtained from the Cobb-Douglas function is not the only one that satisfies the Inada conditions.

13 The special situation obtained in these last examples, namely, that $f^{\prime}(k)$ is discontinuous at $k=1$, and $f^{\prime \prime}(k)$ is continuous at $\mathrm{k}=1$, is a result of the function defined by:

$$
y^{*}=f^{*}(k)=A \sqrt{k}\left(\frac{k^{2}-1}{k^{2}+1}\right), \forall k \in[0, \infty] .
$$

This differs from $y=f(k)$ defined above and has an inflection point at $k=1$. In addition, notice that the function $y=f(k)$ defined above is differentiable $\forall k \in \mathbb{R}^{+}$such that $\{1\}=(0,1) \cup(1, \infty)$ and is not homogeneous to any degree.

\section{Generalization}

EE and Cauchy's condition can be written as a function of any number of variables $Y=F\left(x_{1}, \cdots, x_{n}, L\right)$, namely:

$$
\begin{gathered}
\sum X_{k}=\frac{\partial F}{\partial X_{k}}+L \frac{\partial F}{\partial L}=F, \text { and } \\
\gamma\left(\tau_{1}, \tau_{2}, \cdots, \tau_{n}\right)= \\
F\left(\alpha_{1}\left(\tau_{1}, \cdots, \tau_{n}\right), \cdots, \alpha_{n}\left(\tau_{1}, \cdots, \tau_{n}\right), \beta\left(\tau_{1}, \cdots, \tau_{n}\right)\right)
\end{gathered}
$$

respectively. If the following determinant of the Jacobian matrix differs from zero,

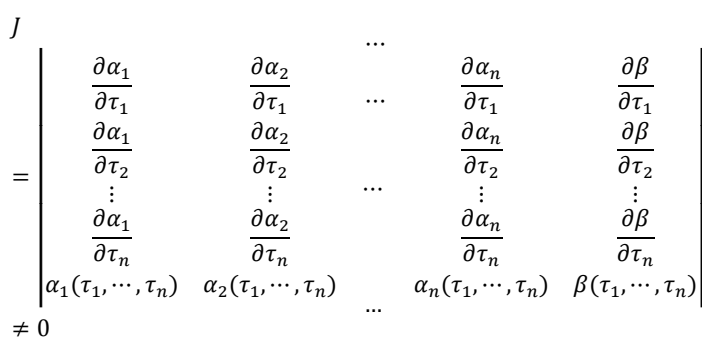

Then, the parametric representation of the unique solution of problems (VI.1) and (VI.2) is:

$\left\{\begin{array}{l}X_{k}=X_{k}\left(\tau_{1}, \tau_{2}, \cdots, \tau_{n}, t\right)=\alpha_{k}\left(\tau_{1}, \tau_{2}, \cdots, \tau_{n}, t\right) e^{t} ; k=1,2, \cdots, n \\ L=L\left(\tau_{1}, \tau_{2}, \cdots, \tau_{n}, t\right)=\beta\left(\tau_{1}, \tau_{2}, \cdots, \tau_{n}, t\right) e^{t} \\ Y=Y\left(\tau_{1}, \tau_{2}, \cdots, \tau_{n}, t\right)=\gamma\left(\tau_{1}, \tau_{2}, \cdots, \tau_{n}, t\right) e^{t}\end{array}\right.$

where $Y=F\left(X_{1}, X_{2}, \cdots, X_{n}, L\right)$

Therefore, all previous results can be generalized to any number of production factors. The empirical problem is now to find the appropriate Cauchy condition; in other words, to find a hypersurface of dimension ' $\mathrm{n}$ ' parametrized by:

$\left\{\begin{array}{l}X_{k}=X_{k}\left(\tau_{1}, \tau_{2}, \cdots, \tau_{n}, 0\right)=\alpha_{k}\left(\tau_{1}, \tau_{2}, \cdots, \tau_{n}\right) ; k=1,2, \cdots, n \\ L=L\left(\tau_{1}, \tau_{2}, \cdots, \tau_{n}, 0\right)=\beta\left(\tau_{1}, \tau_{2}, \cdots, \tau_{n}\right) \\ Y=Y\left(\tau_{1}, \tau_{2}, \cdots, \tau_{n}, 0\right)=\gamma\left(\tau_{1}, \tau_{2}, \cdots, \tau_{n}\right)\end{array}\right.$

such that:

$$
\begin{aligned}
& \gamma\left(\tau_{1}, \tau_{2}, \cdots, \tau_{n}\right) \\
& =F\left(\alpha_{1}\left(\tau_{1}, \cdots, \tau_{n}\right), \cdots, \alpha_{n}\left(\tau_{1}, \cdots, \tau_{n}\right), \beta\left(\tau_{1}, \cdots, \tau_{n}\right)\right)
\end{aligned}
$$

where $Y=F\left(X_{1}, X_{2}, \cdots, X_{n}, L\right)$ (i.e., the characteristic hypersurface of dimension $(n+1): Y=F\left(X_{1}, X_{2}, \cdots, X_{n}, L\right)$ must pass through the given hypersurface of dimension ' $n$ ' (VI.5)). However, these imply additional empirical problems that did not occur in the case of two production factors, namely, we need $\mathrm{n}$ linearly independent parameters $\left(\tau_{1}, \tau_{2}, \cdots, \tau_{n}\right)$, where $\tau_{1}$ can be the time $\tau$. What could be the other $(n-1)$ independent parameters? Mathematically, it may not be very complex to create other $(n-1)$ arbitrary independent parameters, but finding $(n-1)$ independent parameters empirically related to the production of a given activity (i.e., economically significant parameters that allow us to represent the input and output production factors) does not appear to be an easy practical problem. Therefore, if we do not have enough parameters to represent $\left\{X_{k}\right\}_{k=1}^{n}, L$ and $Y$ as 
functions of those parameters, we lose the unicity of the solution of the Cauchy problem for EE, and in general, there are infinite solutions. Hence, we are left with at least three alternatives to finding the production function:

(a) We have $n$ independent parameters $\left\{\tau_{i}\right\}_{i=1}^{n}$ to represent every factor of production $\left\{X_{-} k\right\}_{k=1}^{n}$ and $L$ and the production output $\mathrm{Y}$ on an $\mathrm{n}$-dimensional manifold.

(b) We have continuous differentiable time series in the period of time $[0, T]:\left\{X_{k}(\tau)\right\}_{k=1}^{n} ; L(\tau)$ and $\gamma(\tau)$, obtained from empirical data belonging to the given particular production activity, or

(c) We know that the given production activity follows CRS (i.e., its production function satisfies $\mathrm{EE}$, or it is HDO, which is the same).

The second alternative (b) does not complete the theory nicely because Cauchy's condition reduces to a sort of 'accounting identity', and therefore, we lose the unicity of the solution to problems (VI.1) and (VI.2). Thus, it becomes only a 'heuristic mechanism or methodology' to find the production function. Moreover, because in this case, Cauchy's condition is given on curve $\Gamma$ :

$$
\vec{r}=\vec{r}(\tau)=\left(\alpha_{1}(\tau), \alpha_{2}(\tau), \cdots, \alpha_{n}(\tau), \beta(\tau), \gamma(\tau)\right)
$$

which is obtained from empirical data using any method of adjustment, it can also be applied to industries, sectors, nations, zones, areas, or globally, if we are able to obtain the corresponding empirical index numbers and the curve that adjusts such empirical data, avoiding the 'famous problem of aggregation'.

The third alternative (c) gives rise to the second heuristic mechanism (or methodology) (besides that mentioned in Section IV for which three examples were given), based on the observation that the concept of HDO (or CRS) is equivalent to EE.

However, the first alternative (a) completes the whole theory presented here nicely and completely because we can do everything we did in the case of the two independent and homogeneous production factors. In particular, we obtain the unique solution represented by (VI.4), and we can apply to it. All the advanced integral-differential calculus and differential geometry we need to quantify the properties of the production function are thusly represented. We can impose additional properties on the solution (VI.4), such as the laws of decreasing, constant, or increasing marginal returns, which (similar to the case of two production factors) are expressed in terms of some relations that must satisfy $\alpha_{1}, \alpha_{2}, \cdots, \alpha_{n}, \beta$, and $\gamma$ and their first and second partial derivatives with respect to the parameters $\tau_{1}, \tau_{2}, \cdots, \tau_{n}$. More importantly, we define the production function of the given productive activity as the function $Y=F\left(X_{1}, X_{2}, \cdots, X_{n}, L\right)$ that satisfies the Cauchy problems (VI.1) and (VI.2) or have the form (VI.4), where $\alpha_{1}\left(\tau_{1}, \cdots, \tau_{n}\right), \alpha_{2}\left(\tau_{1}, \cdots, \tau_{n}\right), \cdots, \alpha_{n}\left(\tau_{1}, \cdots, \tau_{n}\right), \beta\left(\tau_{1}, \cdots, \tau_{n}\right)$ is the parametric representation of the n-dimensional manifold data associated with the given particular production activity that satisfies condition (VI.3). Therefore, in this case (as in the case of the two production factors), the theory is nicely completed. In other words, problems (VI.1) and (VI.2) have a solution that is unique and depends continuously on the Cauchy data condition. Moreover, because the Jacobian matrix (VI.3) differs from zero, it follows from the inverse function theorem that the transformation:

$$
\left\{\begin{array}{l}
X_{k}=\alpha_{k}\left(\tau_{1}, \tau_{2}, \cdots, \tau_{n}\right) e^{t} ; k=1,2, \cdots, n \\
L=\beta\left(\tau_{1}, \tau_{2}, \cdots, \tau_{n}\right) e^{t}
\end{array}\right.
$$

is locally bijective (i.e., it is locally invertible). Therefore, (VI.3) guarantees that locally (VI.4) represents a hypersurface $\wedge$ of the form:

$$
Y=F\left(X_{1}, X_{2}, \cdots, X_{n}, L\right)
$$

where (VI.4) is the parametric representation of the same hypersurface $\Sigma$.

Expressions (VI.4) and (VI.8) are two different forms representing the corresponding unique production function, to which we can apply all the needed advanced calculus and differential geometry to quantify its properties. Furthermore, (VI.5) is a manifold obtained from the empirical data of the given production activity. However, as mentioned before, obtaining the manifold (VI.5) from empirical data using some hypersurface adjustment technique (such as the square minimum) presents two difficulties. The first is to obtain ' $n$ ' measurable economically significant independent parameters, in terms of which we can represent the output and production factors, and the second is to have enough empirical data represented in these forms to obtain the manifold (VI.5) from the empirical data using a hypersurface adjustment technique. These difficulties make it very challenging to apply these theories from a practical point of view.

\section{Conclusions}

Using elements of the theory of partial differential equations of the first order, such as EE and Cauchy's condition, we have developed basic mathematical tools to build a theory to produce neoclassical production functions. By further imposing the condition that the determinant of the Jacobian matrix $\mathrm{J} 6=0$, we obtain a unique parametric representation of a function that satisfies, first, the CRS property that depends on the initial data involved in Cauchy's condition. We refer to this preliminary function as a protoproduction function. Then, we deduce the conditions that the initial data must satisfy to ensure that this function complies with the law of diminishing marginal returns. In this context, we mention that the initial data involved in Cauchy's condition can be interpreted (in the case of a two-factor production function) as time series data obtained from empirical data associated with a given economic activity. Therefore, Cauchy's condition is a relevant part of our theory because it combines our theoretical model with the empirical knowledge available for a given economic activity. From this strategy, the 
complex problem of aggregation found in macroeconomics depends now on the possibility of obtaining the corresponding initial data and the ability to adjust a hypersurface to such data (using some numerical adjusting technique such as square minimums), which represents Cauchy's condition for this problem. Additionally, our theory integrates the mathematically necessary conditions for representing the production function in per capita terms, at least for two production factors. Considering all these results, we think that, from a purely theoretical point of view, our theory is complete. However, from a practical point of view, except in the case of only two production factors, there are serious empirical difficulties to be overcome. As a final comment, we claim that at the current state of our research, it is impossible to know if our theory will overcome the problems pointed out by the heterodox side of the Cambridge controversy mentioned in Section I, which predicts the impossibility of building production functions because it is not possible to construct a quantity such as an "aggregate capital stock" that could be embedded in an "aggregate production function" in any meaningful way. This will be one of the central focuses of our future research agenda. ${ }^{14}$

\section{Acknowledgments}

The authors are grateful for the comments and changes proposed by the anonymous referees who reviewed this paper. Orellana and Fuentes are also grateful for the financial support provided by the FONDECYT 1181414 project - "Análisis Crítico de Algunos Modelos Matemáticos en Economía"- of the Agencia Nacional de Investigación y Desaroollo del Gobierno de Chile (National Agency for Research and Development of the Government of Chile). They are also grateful for the sponsorship of the Dirección General de Investigación, Innovación y Posgrado, and the Departments of Mathematics and Industry of the Universidad Técnica Federico Santa María.

\section{REFERENCES}

[1] Arrow K., Chenery H., Minhas B. and R. Solow, "Capital-Labor Substitution and Economic Efficiency", The Review of Economic and Statistics, Vol XLIII, (3), 1961, pp: 225-250.

[2] Barro R. and Sala-i-Marti X., Economic Growth, The MIT Press, Second Edition, 2003.

[3] Biddle JE, "Progress through Regression: The Life Story of

14 According to our theory, the critique raised by Shaik (1974) and his followers in relation to the Cobb-Douglas and CES production functions is located at the level of Cauchy's condition. In fact, he showed that the Cobb-Douglas production function of the type $\gamma(\tau)=A \alpha^{m}(\tau) \beta^{1-m}(\tau)$ can be easily deduced from the accounting identity $\gamma(\tau)=r \alpha(\tau)+$ $w \beta(\tau)$. However, neither of these two relations are production functions; they are only two apparently different relations based on empirical data given in the form of time series $\alpha(\tau), \beta(\tau), \gamma(\tau)$ associated with capital $K$, labor $L$, and output $Y$. the Empirical Cobb-Douglas Production Function (Historical Perspectives on Modern Economics)", Cambridge University Press, November 2020.

[4] Brand L, "Advanced Calculus", John Wisley and Sons Publisher, 1983.

[5] Budak B.M. and S.V. Fomin, "An Advanced Course in Higher Mathematics”, Mir Publishers, 1973.

[6] Cobb, C.W. and Douglas, P.H., "A Theory of Production. American Economic Review”, 18, 1928, pp.: 139-165.

[7] Felipe, J. and J.S.L McCombie, "The Aggregate Production Function: 'Not Even Wrong"', Review of Political Economy, Volume 26, Issue 1, 2014.

[8] Garabedian, Paul, "Partial Differential Equations", American Mathematical Society, 1964.

[9] Hadamard, Jacques, "Sur les problèmes aux dérivéees partielles et leur signification physique". Princeton University Bulletin, 1902, pp. 49-52.

[10] Karagiannis G., Palivos T. and C. Papageorgiou. 2005, "Variable Elasticity of Substitution and Economic Growth: Theory and Evidence", In New Trends in Macroeconomics, Edited by Diebolt Claude and C. Kyrtsou Catherine. Berlin/Heidelberg: Springer, 2005, pp. 21-37.

[11] Krugman P. and Obstfeld M., International Economics: Theory and Practice, Addison Wesley, $6^{\text {th }}$ Edition, 2002.

[12] Manfreds P. Do Carmo, "Geometría Diferencial de Curvas y Superficies”, Alianza Editorial, 1995.

[13] Mas-Colell A., Whinston M. and Green J., Microeconomic Theory, Oxford University Press, 1995.

[14] Mishra SE, "A Brief History of Production Functions", MPRA Paper 5254, University Library of Munich, Germany, 2007.

[15] Revankar, Nagesh S. 1971. "A class of variable elasticity of substitution production functions, Econometrica 39, 1971, pp.: 61-71.

[16] Robinson J., "The Production Function and the Theory of Capital", The Review of Economic Studies, Vol. 21, No. 2 (1953 -1954), 1957, pp. 81-106.

[17] Shaik A., "Laws of Algebra and Laws of Production: The Humbug Production Function". The Review of Economics and Statistics, Vol LXI, No 1, February 1974.

[18] Thach NN, "Macroeconomic Growth in Vietnam Transitioned to Market: An Unrestricted VES Framework", Economies 2020, 8, 58; pp.: 8-15.

[19] Thomas George B. and Ross L. Finey, "Calculus and Analytic Geometry", Addison-Wisley Publishing Company, 1992.

[20] Vilcu. G.E, "A geometric perspective on the generalized Cobb-Douglas production functions", Applied Mathematics Letters, vol. 24, no. 5, 2011, pp. 777-783.

[21] Vilcu. A.D. and G.E., "On some geometric properties of the generalized CES production functions", Applied Mathematics and Computation, vol. 218, no. 1, 2011, pp. 124-129.

[22] Zauderer, Erich, "Partial Differential Equations of Applied Mathematics", John Wisley and Sons Publisher, 1983. 